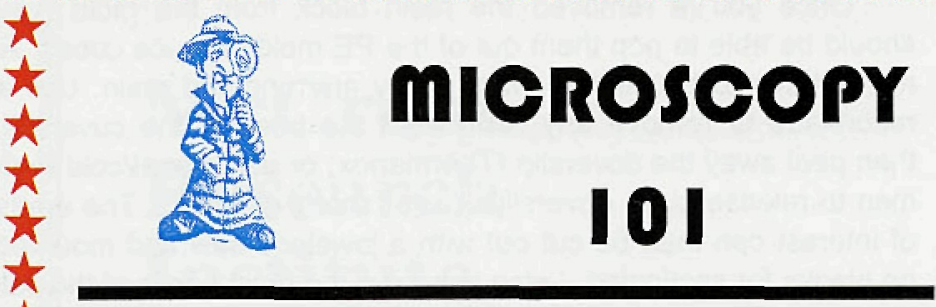

We appreciate the response to this publication feature - and welcome all contributions. Contributions may be sent to Phil Oshel, our Technical Editor at:

Mr. Phil Oshel Tel.: (608)833-2885

PO Box $620068 \quad$ Fax: (608)836-1969

Middleton W1 53562 eMail: peoshel@facstaff.wisc.edu

\section{Resolution and Resolving Power}

A very basic point that is almost always overlooked when discussing SEM "resolution," is the difference between "resolution" and "resolving power."

An SEM might have a resolving power of $1 \mathrm{~nm}$ or so based on the observation of gaps between gold particles on a featureless carbon film. Fine--if ones job is to look at gold on $\mathrm{C}$.

For the actual 3-D samples most everyone looks at, EVERY micrograph has a DIFFERENT resolution, closer to $10 \mathrm{~nm}$, because of secondaries generated by back scattered electrons, edge effects, ...etc. If you want resolutions closer to the instrument's resolving power you have to look at thin TEM specimens without sharp edges and angles.

In our semiconductor lab we have several FESEMs advertised to have resolutions of $1 \mathrm{~nm}$ that can not resolve 4 and $5 \mathrm{~nm}$ layers in a film stack. There is no question that these instruments are superior to non-FESEMs and that today's SEMs are feature-filled and wonderfully reliable--just don't expect $1 \mathrm{~nm}$ resolution in real images and remember the difference between resollution and resolving power.

So if every instrument has one "resolving power" and a different "resolution" for each picture, the only test of "resolution" is to take ones bread-and-butter samples to each manufacturer and compare the resulting images. There is not any theoretical formula that will provide a more meaningful answer.

\section{Ron Anderson, IBM Corporation anderron@us.ibm.com}

\section{Safe Disposal of Mercury Salts}

Make sure that gloves are worn for this procedure. All containers and gloves used should be rinsed with water and the water put in the waste mercury container for next time.

All solutions containing mercury salts are collected into a jar labelled "Used Mercury Salts". We keep this in our grossing room. The jar is actually a clear glass $3 \mathrm{~kg}$ sodium phosphate container.

When the jar is about half full, to each $900 \mathrm{~mL}$ (estimate, don't measure) add 40 grams of sodium carbonate to raise the $\mathrm{pH}$ to 8.0 or higher and mix well.

Leave it for a while, then filter the solution and collect the precipitate into a plastic jar for disposal. Put the filter paper in the jar as well. Seal the jar very well. We coat the lid with several layers of paraffin wax until it is about a quarter inch thick. Rinse the funnel and put the washings into the waste mercury container.

The filtrate should be clear. If it is not, repeat the process. We check by adding a little dissolved sodium carbonate to see if any more precipitate forms. If it does not, all mercury salts have been removed and the filtrate may safely be disposed of down the sink if local regulations allow.

Pack the jar containing the precipitate inside a safety container and seal.

This procedure at the least works with B5, formol sublimate and similar fixatives. In practice these are the two biggest problems with disposal. It should work with mercury-dichromate mixtures (Zenker type), but I have no idea what the reaction is with the dichromate.

Reference:

Crookham, J \& R Dapson. Hazardous Chemicals in the Histopathology Laboratory. Anatech, Ltd.

$$
\begin{aligned}
& \text { Bryan D. Llewellyn, } \\
& \begin{array}{c}
\text { Prince George Regional Hospital, British Columbia } \\
\text { bryand@netbistro.com }
\end{array}
\end{aligned}
$$

\section{Cleaning Platinum Crucibles}

Many years ago we regularly used platinum crucibles in many analytical chemistry procedures, and according to my analytical chemistry book, platinum can be cleaned by fusion with potassium or sodium bisulfite. I later adopted this procedure with good success to clean the platinum apertures for an RCA EML electron microscope.

Get a small ceramic crucible, put a bit of the bisulfite in it, heat it until the bisulfite melts, then drop the apertures into the melt. After a few minutes allow the melt to solidify, dissolve the bisulfite with hot water, and your apertures should be as bright and shiny as new.

If this does not work, try heating them in a mixture of equal parts of concentrated hydrofluoric acid and concentrated hydrochloric acid - using appropriate safety precautions, of course!

\section{Wilbur C. Bigelow, University of Michigan bigelow@umich.edu}

\section{Preparing Diatoms for EM}

Diatoms make for beautiful specimens for both transmission and scanning electron microscopy. As well, they are studied by many people, and there is always a need for good diatom preparations for EM.

I find that the diatoms in diatomaceous earth are usually broken, and like to prepare my own from fresh specimens. The materials needed for this are a plankton net, some potassium dichromate, and 30\% hydrogen peroxide. Once the diatoms are collected (plankton net tows from shoreline, or wherever), they can be cleaned using the chemicals.

1) Place the diatoms in a large beaker (and I mean large here) then pour in 15 to $20 \mathrm{~mL}$ of hydrogen peroxide followed by a large pinch of the potasium dichromate. The resultant reaction oxidizes the organics, leaving the glass frustules of the diatoms unharmed. It is better to be cautious with the amounts used as this is an exothermic reaction, sometimes violently so. Not that there will be an 